\title{
КОСВЕННОЕ ЭКСПЕРИМЕНТАЛЬНОЕ ОПРЕДЕЛЕНИЕ ПЕРЕДАТОЧНЫХ ФУНКЦИЙ ПРЕОБРАЗОВАТЕЛЕЙ ЭЛЕКТРОЭНЕРГИИ
}

R. ALLIKAS. MUUNDURITE OLEKANDEFUNKTSIOONIDE KAUDNE EKSPERIMENTAALNE MAÄRAMINE

R. ALLIKAS. INDIRECT EXPERIMENTAL DETERMINATION OF THE TRANSFER FUNCTION OF THE POWER CONVERTER

(Представил И. Эпик)

Рассмотрим обычную ситуацию: имеется работающий преобразователь параметров электроэнергии или его макет, а характеристики нагрузки определены заранее теоретически или экспериментально. Требуется исследовать совмещение работы данного преобразователя с заданной нагрузкой. Обычно по известным характеристикам нагрузки строится соответствуюший макет и необходимые исследования физических процессов проводятся экспериментальным путем. Но иногда построение макета нагрузки или осуществление совместной работы преобразователя с нагрузкой связано с трудностями.

Приведем два примера. Во-первых, дуговые нагрузки имеют отрицательное динамическое сопротивление. Трудность заключается в совмещении работы управляемого выпрямителя или широтно-импульсного модулятора с фильтром и с дуговой нагрузкой на реальных макетах. Во-вторых, соленоиды для создания сверхсильных магнитных полей имеют частотную характеристику, отличную от таковой обыкновенных индуктивностей (наклон менее $20 \partial Б$ в диапазоне высоких частот). Построение макета нагрузки с такой характеристикой также связано со сложностями. Для решения таких задач предложено использовать следующую методику.

Сначала определяется математическая модель исследуемого преобразователя, а затем вычисляются на ее основе необходимые фнзнческие процессы и характеристики с учетом заданных или заранее определенных характеристик нагрузок.

Рассмотрим случай, когда требуется исследовать регулировочные свойства (соответствующие частотные характеристики) компенсационного устройства, частота работы которого приблизительно в десять раз больше частоты коммутации вентилей управляемого выпрямителя. Такое ограничение частотного диапазона позволяет применять линейную математическую модель для анализа физических процессов в преобразователе. Динамическое сопротивление дуги вблизи рабочей точки аппроксимируется тоже линейным отрицательным сопротивлением.

Составим систему уравнений, описывающую процессы в исследуемом преобразователе. Решая эту систему в отношении выходного тока $i k$ c учетом комплексных сопротивлений в ветвях, получим 


$$
i k=T 3 * e /\left(T 1^{\prime}+T 2^{\prime} * z k\right),
$$

где $e$ - выходное напряжение (эдс); $z k-$ комплексное сопротивление выходной ветви; $T 2^{\prime}-$ сумма миноров, соответствующая $z k ; T 1^{\prime}-$ остальная часть детерминанта системы.

После деления числителя и знаменателя дроби уравнения (1) на комплексное число $T 3 \neq 0$ получим

$$
20 \lg (i k / e)=20 \lg (1 /(T 1+T 2 * z k)) .
$$

Для нахождения передаточных функций преобразователя необходимо знать величины $T 1$ и $T 2$. Эти, от частоты зависящие комплексные числа, удобно определить из эксперимента. Для этого необходимо иметь по меньшей мере два разных макета нагрузки, частотные характеристики которых известны. Проводится два эксперимента с этими нагрузками и вычисляются величины $T 1$ и $T 2$ из системы уравнений (4). В эксперименте сначала регистрируется временный ход входного и выходного сигналов (напряжения или тока), а затем находятся их гармонические. Эксперимент можно провести и классически - по отдельным гармоникам. Для вычисления $T 1$ и $T 2$ получим

$$
\left|\begin{array}{ll}
1 & z k 1 \\
1 & z k 2
\end{array}\right| *\left|\begin{array}{l}
T 1 \\
T 2
\end{array}\right|=\left|\begin{array}{c}
e 1 / i k 1 \\
e 2 / i k 2
\end{array}\right| \text {, }
$$

откуда

$$
\begin{aligned}
& T 1=\frac{1}{D}\left|\begin{array}{ll}
1 & e 1 / i k 1 \\
1 & e 1 / i k 2
\end{array}\right|, \quad D=\left|\begin{array}{ll}
1 & z k 1 \\
1 & z k 2
\end{array}\right|, \\
& T 2=\frac{1}{D}\left|\begin{array}{ll}
e 1 / i k 1 & 1 \\
e 2 / i k 2 & 1
\end{array}\right|,
\end{aligned}
$$

где $z k 1, z k 2$ - комплексные сопротивления известных нагрузок.

В качестве простого примера выпишем уравнения топологической схемы, приведенной на рисунке.

Предположим, что эдс $e$ является входным сигналом, а ток в нагрузке $z k-$ выходным. Для выходного тока получим (см. уравнение (1))

$$
\begin{gathered}
T 1^{\prime}=\left|\begin{array}{rrrrr}
1 & -1 & -1 & 0 & 0 \\
0 & 0 & 1 & -1 & -1 \\
0 & 0 & 0 & -z 4 & z k \\
0 & -z 2 & z 3 & z 4 & 0 \\
z 1 & z 2 & 0 & 0 & 0
\end{array}\right| *\left|\begin{array}{l}
i 1 \\
i 2 \\
i 3 \\
i 4 \\
i k
\end{array}\right|=\left|\begin{array}{l}
0 \\
0 \\
0 \\
0 \\
e
\end{array}\right|, \\
0 \begin{array}{rrrr}
1 & -1 & -1 & 0 \\
0 & 0 & 1 & -1 \\
0 & -z 2 & z 3 & z 4 \\
z 1 & z 2 & 0 & 0
\end{array}\left|, \quad T 2^{\prime}=\right| \begin{array}{rrrr}
1 & -1 & -1 & 0 \\
0 & 0 & 0 & -z 4 \\
0 & -z 2 & z 3 & z 4 \\
z 1 & z 2 & 0 & 0
\end{array} \mid, \\
T 3=\left|\begin{array}{rrrr}
1 & -1 & -1 & 0 \\
0 & 0 & 1 & -1 \\
0 & 0 & 0 & z 4 \\
0 & -z 2 & z 3 & z 4
\end{array}\right| .
\end{gathered}
$$




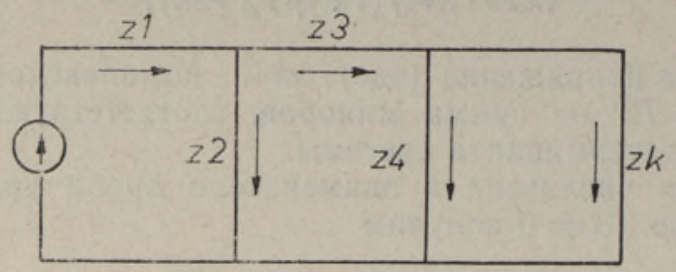

Из уравнений (6) определяются величины $T 1^{\prime}, T 2^{\prime}$ и $T 3$ (см. уравнение (1)), которые далее преобразуются по изложенной выше методике. Отметим, что с помощью уравнений типа (1) и (6) удается ускорить процесс вычислений, если комплексные сопротивления $(z 1, z 2$, $z 3, z 4)$ заданы, а переходные характеристики необходимо рассчитывать для разных нагрузок.

Искомую переходную частотную характеристику можно определить при необходимости и в кусочно-линейном приближении, используя для этого несколько известных пар нагрузок и те частотные области, где выполняется условие линейности модели. Независимость величин $T 1$ и $T 2$ от амплитуды входного сигнала и от выбора разных пар известных экспериментальных нагрузок характеризует линейность найденной математической модели преобразователя в данной частотной области.

Полученные из эксперимента временные кривые входного и выходного сигналов можно вводить непосредственно в ЭВМ, предварительно преобразовав их в дискретный код. Если входные и выходные величины содержат достаточное количество гармонических, амплитудные и фазовые соотношения между входными и выходными гармониками определяются с помощью быстрого преобразования Фурье [ $\left.{ }^{1}\right]$. По этим соотношениям строятся соответствующие частотные характеристики преобразователя.

В рассматриваемых случаях число параметров математической модели ( $T 1$ и $T 2)$, определенных из эксперимента, не зависит от сложности топологической схемы преобразователя с фильтром. Используя их, удается косвенно, на уровне математической модели, определить физические процессы и передаточные функции в макетах преобразователей, минуя весьма трудоемкую операцию совмещения работы преобразователя с нагрузкой или построение сложных макетов некоторых типов нагрузок.

\section{Л И Т Р А Т У Р А}

1. Р а б ин ер Л., Гоулд Б., Теория и применение цифровой обработки сигналов, М., «Мир», 1978.

Институт термофизики и электрофизики Академии наук Эстонской ССР

Поступила в редакцию $27 /$ IV 1981 